\title{
Influence of Body Mass Index on TREATMENT of BREATHING-Related SLEEP DISORDERS
}

\author{
E. Dzieciolowska-Baran¹,2, A. Gawlikowska-Sroka², I. Poziomkowska-Gesicka³, I. Teul-Swiniarska², \\ T. Sroczynski ${ }^{4}$ \\ ${ }^{1}$ Department of Otolaryngology, Independent Provincial Hospital, Szczecin, Poland; ${ }^{2}$ Department of General and Clinical Anatomy, \\ ${ }^{3}$ Department of Clinical Allergology, and ${ }^{4}$ Department of Physiology, Pomeranian Medical University, Szczecin, Poland
}

\begin{abstract}
Background: Breathing-related sleep disorders cover several conditions (isolated snoring, UARS - upper airway resistance syndrome, obstructive sleep apnea, hypopnea, obesity hypoventilation syndrome) characterized by a variety of symptoms and complex etiology. The conditions can be successfully treated in most cases. Excessive body mass is a factor increasing the probability of the disorders. In most patients it is the only reason for breathing-related sleep disorders. However, it often coexists with various anatomical abnormalities in the upper airway, endocrinological diseases or genetic defects of the facial skeleton, and occurs more frequently in older people, especially men. Excessive body mass significantly affects the range and success of the treatment.

Objective: To analyze treatment outcome in patients treated at the otolaryngology unit for snoring and related diseases with submucosal tissue reduction within the nasal cavity, pharynx, and soft palate.

Material and methods: Patients were stratified into three study groups depending on the body mass index (BMI): normal, overweight, and obese. The BMI value was compared to the severity of breathing disorders during sleep, with the incidence of other systemic diseases (e.g., hypertension, diabetes), and with treatment outcome.

Results and conclusions: The analysis demonstrated a significant influence of body mass on snoring, particularly in complicated and severe types of breathing disorders, such obstructive sleep apnea or hypopnea, and the obesity hypoventilation syndrome. Corrective interventions carried out to eliminate anatomical abnormalities causing obstruction of upper airways provided the best therapeutic effects in patients with normal body mass.
\end{abstract}

Key words: snoring, sleep apnea, BMI, obesity, overweight, treatment

\section{INTRODUCTION}

In recent times there has been a significant increase in interest concerning breathing disorders during sleep among both medical professionals and patients [1-3]. In the case of the latter group, the rising interest could result from better access to information, and thus the awareness of the possible consequences of ignoring the problem [3]. Large, multicenter studies have confirmed that breathing problems during sleep may cause or exacerbate existing symptoms, e.g., hypertension, pulmonary hypertension, ischemic heart disease increased risk of heart attack, cardiac arrhythmia, heart failure, abnormal cerebral circulation - increased risk of stroke, glucose intolerance, diabetes, metabolic syndrome, impaired concentration, depression, and impotence [1].

At the same time, continuous improvement in the standard of living creates an opportunity and, to some degree, forces some people to improve many aspects of their everyday existence, which until now have been neglected or treated marginally. These are hygiene, quality and quantity of rest, including sleep. Thus there are tendencies to try to eliminate one of the fundamental and probably the most spectacular symptoms indicating the irregularity of breathing during sleep, namely snoring. This symptom may significantly affect the relationship with the partner, members of the household, or a travel companion [4].

Unfortunately, together with the improvement of living conditions the incidence of society-related diseases increases [2]. Easy access to food, increase in its energy value and increased consumption all lead to obesity. By definition, it is the accumulation of body fat in excess of physiological needs and adaptive capacity $[5,6]$. Obesity is considered a condition in which fat accounts for more than $20 \%$ of total body mass in men and $25 \%$ in women. For adults with average muscle conditions, the BMI is reflected in the content of body fat. The value of a normal BMI is 19-25, with 25-30 considered overweight, while obesity is $>30$, and $>40$ indicates extreme obesity [7]. Overfeeding in conjunction with a significant decrease in energy expenditure associated with daily activities as a result of technological progress, is now the main cause of a large percentage of people with excessive body weight in fast-developing societies [2, 6].

The phenomenon appears to be a vicious circle. Obesity induces abnormal breathing during sleep, leading to health deterioration in patients with cardiac diseases (also reinforced by increased BMI), which then leads to permanent fatigue, which in turn limits 
the opportunities for physical activity. Lack of exercise increases obesity $[8,9]$. There may be some mood disorders, including depression. Sick people learn that eating can reduce the symptoms of depression in part by providing a short-term pleasure $[10,11]$.

Snoring occurs because of blocking of the flow of air through the respiratory system. This leads to the formation of turbulence and vibrations of the soft palate and uvula. There are many reasons for this hampering of the flow of air. Obesity can be caused primarily by the pressure of fat on the throat and weakening of the pharynx muscle [12]. Endocrine disorders may also be important, often coexisting with obesity, and especially diabetes mellitus (the less common endocrine disorders include hypothyroidism, Cushing's syndrome, polycystic ovary syndrome, and growth hormone deficiency).

Among disordered breathing during sleep, for clinical purposes, the following can be distinguished: isolated snoring, upper airway resistance syndrome (UARS), apnea or hypopnea during sleep, and hypoxia associated with obesity (hypoventilation syndrome). The term snoring is used to describe an acoustic phenomenon during sleep developing as a result of vibrations of some structures within the respiratory system. Upper airway resistance syndrome (UARS) is characterized by recurrent snoring episodes with increasing intensity (crescendo snoring), interrupted by awakenings during which the flow of air through the upper airway returns temporarily to a normal condition. This syndrome occurs more frequently in women suffering from anxiety states. In anamnesis, patients frequently report an accompanying sensation of sleepiness and fatigue during daytime. Apnea is a term used to name the recurring suspension of breathing during sleep, lasting longer than 10 seconds. The degree of sleep apnea is described by the number of episodes per hour of sleep: normal $<5$; $\mathrm{I}^{\circ}$ mild 5-15; $\mathrm{II}^{\mathrm{O}}$ moderate $16-30$; and $\mathrm{III}^{\mathrm{O}}$ severe $>30$. There is a distinction between obstructive sleep apnea (OSA), consisting in falling back of the upper airway walls (most frequent), and the rarely met central sleep apnea, which is related to respiratory reflex blockade. There may also be a mixed sleep apnea. Sleep apnea usually coexists with snoring. Obesity hypoventilation syndrome is defined as the combination of obesity (BMI >30), hypoxia during sleep, and hy- percapnia during the day, resulting from hypoventilation (excessively slow or shallow breathing). The disease has been known since the 1950s, initially as 'Pickwickian syndrome', in reference to a Dickensian character, but currently under a more descriptive name [13].

\section{MATERIAL AND METHODS}

The study was performed in accord with the Declaration of Helsinki for Human Research and the study protocol was approved by an institutional Ethics Committee.

The study material consisted of patients treated surgically at the Department of Otolaryngology, of the Provincial Hospital in Szczecin, in 2009-2010 due to disordered breathing during sleep in the form of snoring, with or without sleep apnea. The treatment goal was to restore the proper passage of air through the upper respiratory tract. The study included only cases in which primary surgery was performed to reduce soft tissue by submucosal resection using the RaVoR technique. Using this method, lower nasal turbinate reductions (mucotomy - Procedure 1 in the tables below)) and/or uvulopalatopharyngoplasty (UPPP - Procedure 2 in the tables below) were performed, in some patients by additional simultaneous surgical procedures such as septoplasty or tonsillectomy. 49 patients (10 women and 39 men), divided with respect to $\mathrm{BMI}$ into 3 groups:

- Normal weight $(\mathrm{BMI}<25)$

- Overweight (BMI 25-30)

- Obesity (BMI >30)

The patients' main complaints were defined as sleep apnea or simple snoring (with/without the combination of increased upper airway resistance during sleep).

We analyzed the correlation between BMI and age of patients and the severity of breathing disorders during sleep, and pre-evaluated BMI impact on the laryngological treatment outcomes. Despite a considerable difference in size between the groups of men and women, we also evaluated the results in relation to gender, and analyzed the coexistence of other systemic diseases, such as hypertension and diabetes.

Table 1. List of procedures to improve airway patency performed in men/women (M/F) with normal, overweight, and obese BMI at the Department of Otolaryngology of the Provincial Hospital in Szczecin (2009-2010).

\begin{tabular}{lcccc}
\hline Type of treatment & $\begin{array}{c}\text { Number of patients } \\
\mathbf{M} / \mathbf{F}\end{array}$ & $\begin{array}{c}\mathbf{B M I}<\mathbf{2 5} \\
\mathbf{M} / \mathbf{F}\end{array}$ & $\begin{array}{c}\text { BMI 25-29 } \\
\mathbf{M} / \mathbf{F}\end{array}$ & $\begin{array}{c}\text { BMI }>\mathbf{3 0} \\
\mathbf{M} / \mathbf{F}\end{array}$ \\
\hline RaVoR-mucotomy (1) & $4 / 6$ & $2 / 4$ & $2 / 0$ & $0 / 2$ \\
RaVoR-UPPP (2) & $7 / 0$ & $0 / 0$ & $2 / 0$ & $5 / 0$ \\
RaVoR 1+2 & $8 / 1$ & $0 / 0$ & $1 / 0$ & $7 / 1$ \\
RaVoR 1+septoplasty & $18 / 2$ & $5 / 1$ & $8 / 0$ & $5 / 1$ \\
RaVoR 1+tonsillectomy & $0 / 1$ & $0 / 1$ & $0 / 0$ & $0 / 0$ \\
RaVoR 2+tonsillectomy & $2 / 0$ & $0 / 0$ & $1 / 0$ & $1 / 0$ \\
Total & $\mathbf{3 9 / 1 0}$ & $\mathbf{7 / 6}$ & $\mathbf{1 4 / 0}$ & $\mathbf{1 8 / 4}$ \\
\hline
\end{tabular}




\section{RESULTS}

The study group was dominated by men $(80 \%)$, aged 19-71; the age of women $(20 \%)$ ranged from 16 to 59 years. BMI showed an increasing trend with the age of patients; the trend being more pronounced in women (Fig. 1). The majority of males, who were operated on, were obese - $18(46 \%), 14$ were overweight (36\%), and only 7 of them $(18 \%)$ had normal body weight. Six women had normal weight, there were no overweight women, but obesity was found in 4 of them (Table 1).

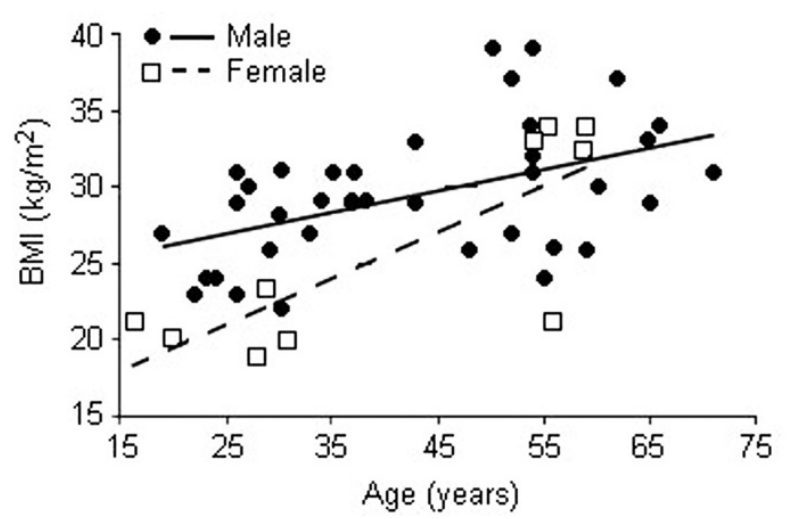

Fig. 1. Comparison of BMI vs. age of patients from a gender perspective.

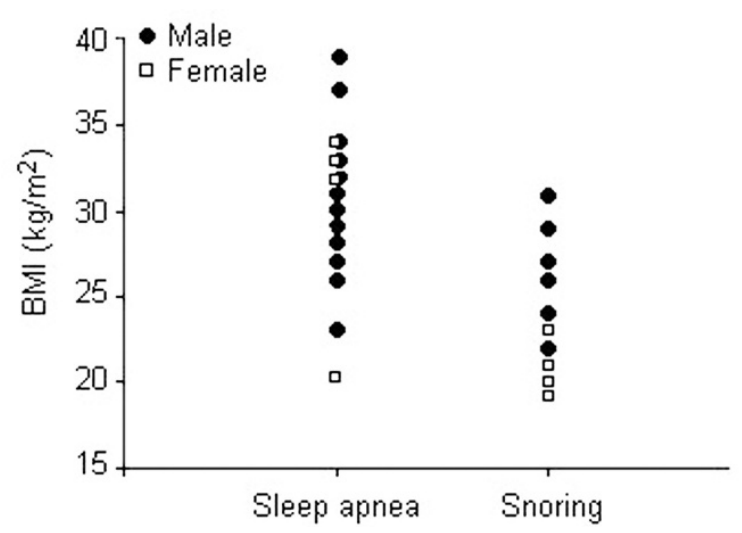

Fig. 2. Comparison of BMI vs. the type (severity) of clinical symptoms from a gender perspective.

The sleep apnea syndrome was diagnosed in $65 \%$ of patients. Among men, 12 patients suffered from sleep apnea $(31 \%)$ and $27(69 \%)$ from simple snoring. The distribution of snoring and sleep apnea was about equal in the women patients. The patients with apnea had a higher BMI than those with snoring (Fig. 2).

An evident improvement after treatment was observed in $27 \%$ of patients, but they were mainly those with normal weight and with simple snoring; the improvement concerned here equally both genders. A moderate improvement was observed in 33\% of patients with BMI lower than 33. In the remaining 30\% of the treated patients, the procedures used did not produce any effect; the latter group covered people with severe obesity (BMI >34) (Fig. 3). The number of symbols in Fig. 2 and Fig. 3 does not correspond to

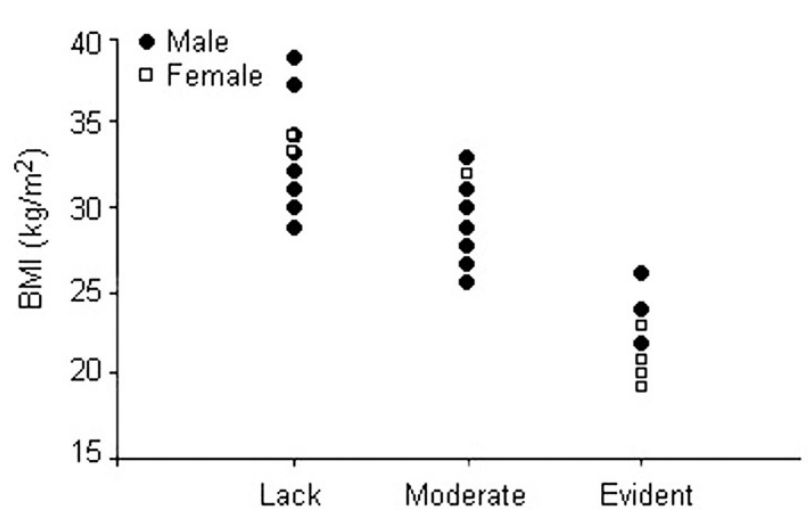

Fig. 3. Comparison of BMI vs. the degree of obtained improvement from a gender perspective.

the number of patients in each group due to a frequent overlap of patients with the same BMI.

The following diseases coexisted in the patients studied: insulin-dependent diabetes (6 persons) and arterial hypertension (8) - among them four patients suffered from both conditions - chronic obstructive pulmonary disease (2), and allergy (7).

\section{DISCUSSION}

The ratio of BMI to age in the study group was fairly typical; it was increasing with aging of patients [14]. The highest values of body weight were recorded in patients older than 55 years and they were found in men, who more often suffer from breathing problems during sleep $[1,2]$. In the group of women - a relatively small group and, unfortunately, not very representative for that reason, age distribution revealed young women with normal weight and suffering from snoring who managed to achieve complete recovery after the applied treatment.

Today, in fast developing countries an alarming trend of increased body weight in younger age groups is observed [15]. Among the group of patients analyzed in this study, the youngest - up to 25 years, in the majority, were of normal weight. Their breathing problems during sleep were confined mainly to simple snoring. It cannot be excluded that these problems were mainly caused by an allergy, which in this group occurred most frequently. Among these patients, treatment results were the best. Allergies can be an isolated risk factor for breathing disorders during sleep. In its progress a partial or complete, temporary or permanent blockage in the upper respiratory tract can occur, especially in the form of nasal allergic rhinitis. Consequently, turbinate hypertrophy develops. The implementation of conservative treatment does not give the expected results because the increased respiratory resistance in the nose forces the patient to change breathing passage [16]. In these cases, it seems reasonable to use the least invasive surgical methods for a reduction of the volume of nasal turbinates [17, 18]. Tissue reduction using radio frequencies is one such method. Simple snoring with mild to moderate apnea is the primary indication for the use of the RaVoR technique $[3,18,19]$. 
The severity of disordered breathing during sleep correlates with increased BMI. Fluctuations in BMI during the life of individuals show changes in the comfort of their everyday life, including sleep and susceptibility to many diseases $[20,21]$. In this study the vast majority of cases of nocturnal apnea were recorded in patients who were overweight, and particularly in those with obesity. There were only two cases of sleep apnea in patients with normal BMI. Particularly noteworthy is the fact that some patients admitted they noticed an increase in snoring and apnea after their body weight increased significantly. Conversely, in several subjects, their partners observed a reduction in snoring intensity and the disappearance or decrease in the number of apnea episodes during the period when they succeeded in reducing body weight.

Unfortunately, obese patients have a great difficulty in acknowledging that their abnormal body weight has an influence on the occurrence of snoring and/or sleep apnea syndrome. They do not accept the fact that a change in eating habits, i.e., a sustained slimming diet is a key element of therapy. In many patients coexisting diseases limit or even prevent the taking of radical, comprehensive surgical treatment, such as surgical uvuloplasty [22]. Diabetes and hypertension often accompany obesity. At the same time breathing disorders during sleep can lead to, or exacerbate, both of these diseases [23, 24].

The proposed method based on the RaVoR technique has some limitations, particularly with respect to effectiveness. A clear lack of improvement is observed in patients suffering from complex forms of sleep apnea. The safety of this method is a supporting fact in undertaking such treatment, for there is no need for prolonged and deep anesthesia, which is extremely important for coexisting hypertension. Low invasiveness is important in the accompanying diabetes, due to minimizing the risk of impaired healing [18].

It appears that this method should be considered early in treatment, preferably with simultaneous treatment for obesity. Even moderate improvement can allow the patient a greater physical activity. An obese patient means a difficult case for a doctor. Complex health problems often have their origins in patients' emotions: lack of acceptance of their appearance, or lack of prospects for a change in the situation, or even a delusive sense of satisfaction and a false belief that obesity is irrelevant here. Therefore, the most logical approach is to encourage the patient to reduce weight in the first place. With proper BMI, the RaVoR method appears to provide ideal results.

Conflicts of interest: No conflicts of interests were declared by the authors in relation to this article.

\section{REFERENCES}

1. Stradling JR, Davis RJ. Sleep 1: Obstructive sleep apnea/hypopnea syndrome: definitions, epidemiology, and natural history. Thorax 2004; 59: 73-8

2. Punjabi NM. The epidemiology of adult obstructive sleep apnea. Proc Am Thorac Soc 2008; 5(2): 136-43.

3. Dzieciolowska-Baran E, Gawlikowska-Sroka A, Czerwinski F. Snoring - the role of the laryngologist in diagnosing and treating its causes. Eur J Med Res 2009; 14 Suppl IV: 67-70.
4. Sharief I, Silva GE, Goodwin JL, Quan SF. Effect of Sleep Disordered Breathing on the Sleep of Bed Partners in the Sleep Heart Health Study. Sleep 2008; 31(10): 1449-56.

5. Lee H, Haris KM, Gordon-Larsen P. Life course perspectives on the links between poverty and obesity during the transition to young adulthood. Popul Res Policy Rev 2009; 28(4): 505-32.

6. Novak M, Ahlgren C, Hammarstrom A. A life-course approach in explaining social inequity in obesity among young adult men and women. Int J Obes (Lond) 2006; 30(1): 191-200.

7. Haslam DW, James WP. Obesity. Lancet 2005; 366 (9492): 1197-209.

8. Okabayashi K, Kasahara E, Uchiyama H, Yokota K, Togashi Y, Kono C, Yamada Y, Amano H, Yamagutchi T, Kawamoto T, Fujihara H, Ueta Y. Obstructive sleep-apnea-hypopnea syndrome patients with overweight and hypertension in Japanese Workplace. J Occup Health 2007; 49: 117-24.

9. Vgontzas AN, Tan TL, Bixler EO, Martin LF, Shubert D, Kales A. Sleep apnea and sleep disruption in obese patients. Arch Intern Med 1994; 154: 1705-11.

10. Millman RP, Fogel BS, McNamara ME, Carlisle CC. Depression as a manifestation of obstructive sleep apnea: reversal with nasal continuous positive airway pressure. J Clin Psychiatry 1989; 50(9): 348-51.

11. Sforza E, de Saint Hilaire Z, Pelissolo A, Rochat T, Ibanez V. Personality, anxiety, and mood traits in patients with sleep-related breathing disorders: effect of reduced daytime alertness. Sleep Med 2002; 3: 139-45.

12. Peppard PE, Young T, Palta M, Dempsey J, Skatrud J. Longitudinal of moderate weight change and sleep-disordered breathing. JAMA 2000; 284: 3015-21.

13. Mokhlesi B, Tulaimat A. Recent advances in obesity hypoventilation syndrome. Chest 2007; 132(4): 132236.

14. Ogden CL, Carroll MD, Curtin LR, McDowell MA, Tabak CJ, Flegal KM. Prevalence of overweight and obesity in the United States, 1999-2004. JAMA 2006; 295(13): 1549-55.

15. Wang Y, Monteiro C, Popkin BM. Trends of obesity and underweight in older children and adolescents in the United States, Brazil, China, and Russia. Am J Clin Nutr 2002; 75: 971-77.

16. Hiraki N, Suzuki H, Udaka T, Shiomori T. Snoring, daytime sleepiness, and nasal obstruction with or without allergic rhinitis. Arch Otolaryngol Head Neck Surg 2008; 134(12): 1254-7.

17. Kizilkaya Z, Ceylan K, Emir H, Yavanoglu A, Unlu I, Samim E, Akagun MC. Comparison of radio frequency tissue volume reduction and submucosal resection with microdebrider in inferior turbinate hypertrophy Otolar Head Neck Surg 2008; 138(2): 176-81.

18. Friedman M, Lin HC, Gurpinar B, Joseph NJ. Minimally invasive single-stage multilevel treatment for obstructive sleep apnea/hypopnea syndrome. Laryngoscope 2007; 117(10): 1859- 63 .

19. Balcerzak J, Karchier E. Radiofrequency termoablation of the soft palate in the treatment of snoring. Otorynolaryng 2007; 6(2): 104-9.

20. Dixon JB, Schachter LM, O’Brien PE. Sleep disturbance and obesity changes following surgically induced weight loss. Arch Intern Med 2001; 161: 102-6.

21. Tuomilehto J, Lindström J, Eriksson JG, Valle TT, Hämäläinen H, Ilanne-Parikka P, Keinänen-Kiukaanniemi S, Laakso M, Louheranta A, Rastas M, Salminen V, Uusitupa M. Finnish Diabetes Prevention Study Group. Prevention of type 2 diabetes mellitus by changes in lifestyle among subjects with impaired glucose tolerance. N Engl J Med 2001; 344(18): 1343-50. 
22. De Berry-Borowiecki B, Kukwa A, Blanks RHI. Indications for palatopharyngoplasty Arch Otolaryngol 1985; 111: 659-63.

23. Lindström J, Ilanne-Parikka P, Peltonen M, Aunola S, Eriksson J, Hemiö K, Hämäläinen H, Härkönen P, Keinänen-Kiukaanniemi S, Laakso M, Louheranta A, Mannelin M, Paturi M, Sundvall J, Valle T, Uusitupa M, Tuomilehto J. Sustained reduction in the incidence of type 2 diabetes by lifestyle intervention: follow-up of the Finnish Diabetes Prevention Study. Lancet 2006; 368(9548): 1673-9.

24. Narkiewicz K. Obesity and hypertension - the issue is more complex than we thought. Nephrol Dial Transplant 2006; 21(2): 264-7.

25. Resta O, Foschino-Barbaro MP, Legari G, Talamo S, Bonfitto P, Palumbo A, Minenna A, Giorgino R, De
Pergola G. Sleep-related breathing disorders, loud snoring and excessive daytime sleepiness in obese subjects. Int J Obes 2001; 25: 669-75.

Address for correspondence:

E. Dzieciolowska-Baran

Department of Anatomy

Pomeranian Medical University

72, Powstancow Wlkp. St.

70-111 Szczecin

Poland

Phone/Fax: + 48914661480

E-mail: edybar@tlen.pl

http://anatomia.pam.szczecin.pl 